\title{
Targeting motor and cognitive networks with multichannel transcranial direct current stimulation along with peripheral stimulation in a subacute stroke survivor: single case study
}

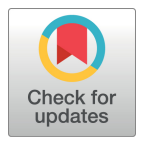

\author{
Divya Midha ${ }^{(1)}$, Narkeesh Arumugam ${ }^{(1)}$ \\ Department of Physiotherapy, Punjabi University, Patiala, India
}

Objective: Reacquisition of motor functions following stroke depends on interhemispheric neural connections. The intervention highlighted in the present case is an insight for augmenting motor recovery by stimulating the lesioned area and adjacent areas governing the motor behaviour of an individual. The purpose of this study was to determine the changes in the motor and cognitive outcomes through multi target stimulation of cortical areas by application of multichannel transcranial direct current stimulation (M-tDCS) in a stroke survivor.

Design: A case report.

Methods: The patient was a participant of a trial registered with the clinical trial registry of India (CTRI/2020/01/022998). The patient was intervened with M-tDCS over the left primary motor cortex i.e. C3 point and left dorsolateral prefrontal cortex i.e. F3 point with 0.5-2 mA intensity for the period of 20 minutes. SaeboFlex-assisted task-oriented training, functional electrical stimulation over the lower extremity (LE) to elicit dorsiflexion at the ankle and eversion of the foot, and conventional physiotherapy rehabilitation including a tailored exercise program were performed. Outcome assessment was done using the Fugl-Meyer assessment scale (FMA) for the upper and lower extremity (UE and LE), Montreal Cognitive Assessment (MOCA), Wisconsin Gait Scale (WGS) and the Stroke Specific Quality of Life (SSQOL) measures. Assessment was taken at Day 0, 15 and 30 post intervention.

Results: Improvement was observed in all the outcome measures i.e FMA (UE and LE), MOCA, SSQOL and WGS across the span of 4 weeks.

Conclusions: M-tDCS induced improvement in motor functions of the UE and LE, gait parameters and cognitive functions of the patient.

Key Words: Brain stimulation, Multichannel transcranial direct current stimulation, Stroke

\section{Introduction}

Stroke is the second leading cause of death [1,2] and third leading cause of disability adjusted life-years across the globe [3]. Stroke is one of the serious public health concerns. Possibly due to substantial increase in the modifiable and non-modifiable risk factors, India stands 2 nd in having the highest rates of mortality and morbidity due to stroke [1]. Stroke affects every domain of an individual varying from motor, sensory, cognitive, perceptual, and psychological domains [4]. Among the constellation of symptoms, motor impairment is the most common, which affects the upper and lower extremities (UEs and LEs) in more than $88 \%$ of stroke victims [5]. Paresis, loss of fine motor skills and movement abnormalities together constitute the upper limb impairments of individuals affected by stroke, which accounts for $73 \%-88 \%$ in first time stroke survivors and $50 \%$ of chronic stroke survivors [6]. Difficulty in walking is another most

Received: 1 September, 2020 Revised: 1 November, 2020 Accepted: 18 November, 2020

Corresponding author: Divya Midha (ORCID https://orcid.org/0000-0002-2198-5553)

Department of Physiotherapy, Punjabi University, NH 64, Urban Estate Phase II, Patiala 147002, India

Tel: 91-8607556615 E-mail: divyamidha.pt@gmail.com

(a) This is an Open-Access article distributed under the terms of the Creative Commons Attribution Non-Commercial License (http://creativecommons.org/licenses/ by-nc/4.0) which permits unrestricted non-commercial use, distribution, and reproduction in any medium, provided the original work is properly cited.

Copyright @ 2020 Korean Academy of Physical Therapy Rehabilitation Science 
common complaint of stroke survivors, making them dependent to a great extent [7].

Persistent disability following stroke impairs activities of daily living performance such as dressing, eating, self-care and personal hygiene etc. which underlies the strong need for the development of a novel neurorehabilitation approach considering the extent of injury and the amount of damage caused by it [8]. Despite the extensive efforts, functional recovery of an individual remains incomplete. Limited understanding of the process of recovery along with the constraints regarding time, lack of cost effectiveness, labour intensive techniques, lack of standardized procedures and lack of long-term adherence to the rehabilitation results in partial recovery $[9,10]$.

Lack of optimal recovery has lead substantial drift of rehabilitation professionals towards the use of technology-aided electrical interventions in the form of transcranial direct current stimulation, utilizing reorganizing capacity of the brain to enhance the motor recovery following stroke. Extensive literature is available on the therapeutic benefits of transcranial direct current stimulation (tDCS) on the motor recovery of stroke. But most trials have targeted single areas of the cortex that were relevant to the specific deficits.

The brain is the hub of cortical connections. The areas of the brain are structurally and functionally related to each other. In the year 1949, Donald Hebb proposed the rule that "Neurons that fire together, wire together". Hebb's rule provided the theoretical base that homosynaptic and heterosynaptic activities facilitate synaptic formation and consolidation during motor rehabilitation [11].

Motor learning entails strong interaction with the cognitive domains like attention, planning, memory and execution control. Hence, the same principle can be applied while stimulating the neuromotor control system at more than one level thereby manipulating the corresponding neuronal circuits and better outcomes may be expected.

Undermentioned is the case of a subacutre stroke survivor intervened with multichannel transcranial direct current stimulation (M-tDCS) along with the peripheral stimulation techniques such as functional electrical stimulation (FES) and exoskeletal device (SaeboFlex; Saebo, Inc., Charlotte, NC, USA) and in which post intervention effects were notified.

\section{Methods}

\section{Case description}

\section{Patient demographics, complaint and history}

A 50-year old male with a history of hypertension and type II diabetes came to the rehabilitation unit with the chief complaint of difficulty in performing activities of daily living and walking. Care givers of the patient reported his ease in forgetfulness of things and difficulty in recall of the things and experiences related to him. The patient had no significant tobacco, alcohol, or recreational drug use history. His past medical history was significant for peripheral neuropathy affecting his feet. The patient was conscious during the episode. There was no history of headache and vomiting and fall associated with episode of stroke. Within two hours on the day of episode, patient noticed clumsiness in his right hand which was soon followed by dragging of his right foot while walking. Within the next hour, slight drooping of the right side of his face and slurring of speech was also noticed and so the patient was admitted for medical management. No family history of previous episodes of stroke is associated with the patient. Computed tomography scans of the patient reflected hypodensity in the middle cerebral artery region and no signs of hemorrhage. Magnetic resonance imaging findings confirmed the diagnosis of ischemic infarct in the left middle cerebral artery territory.

\section{Initial patient screening}

Initial screening of the patient was done with a comprehensive neurological assessment format enclosing a detailed history of the patient, higher mental function assessment, motor examination, reflex examination, sensory assessment, cranial nerve assessment and gait examination with the aim to rule any absolute contraindications for tDCS and other therapeutic interventions.

The patient was oriented to person, place and time. His cognitive screening was done with the Mini-mental status examination (MMSE). His MMSE score was 18 depicting mild cognitive impairment. His spasticity was graded as $1+$ for the right UE and LE, measured on the modified Ashworth scale, and his reflex assessment revealed exaggerated responses. No sensory deficits were found other than lack of propioceptive response. Motor examination revealed that the patient had preserved range of motion of approximately 10 degrees at the wrist joint. His facial nerve was found to be affected along with some hearing deficit on the paralytic side. Patient was ambulatory and was walking 
with the help of a walker. The patient had right facial droop, dysarthria and mild dysphagia.

\section{Intervention}

\section{Criteria for participant selection}

The current patient was the participant of the trial (CTRI/2020/01/022998). Participant selection was done as per selection criteria of the trial. Inclusion criteria was male or female subacute stroke survivors within the age group of 50 to 80 years, Spasticity in the upper or lower extremities having a modified Ashworth scale score $<2$, those with preserved range of wrist extension ( $\approx 10$ degrees), are able to walk, and individuals with mild cognitive impairment with a MMSE score of 18-23, and those willing to participate in the study. The exclusion criteria was individuals diagnosed with hemorrhagic stroke, with a history of neurological disease other than stroke, with a history of musculoskeletal injury/disease affecting the UE and LE, with the history of psychosomatic illness/disease, individuals having cardiovascular \& respiratory problems, any systemic illness, individuals with metallic implants, individuals with a visual analog scale score of $>4$ for pain in the UE/LE, individuals participating in other pharmacological \& rehabilitation studies during the study period, individuals with any sensory problems, or non-cooperative individuals.

\section{Ethics and participant consent}

The patient was a participant of this trial approved by Institutional ethics committee of Punjabi University (IRB No. 152/IEC-2019) and has also been registered with clinical trial registry of India (CTRI/2020/01/022998). An information sheet was given to the patient in which he was notified regarding objectives, procedure, interventions, potential risks and/or expected benefits of the intervention. A written consent was obtained prior to the intervention. The patient was ensured that his identity would be concealed.

\section{Objective assessment}

Baseline assessment was performed using the Fugl-Meyer assessment scale for the upper extremity and lower extremity FMA (UE and LE). The FMA is a multi-item, performance-based impairment index which evaluates balance, sensation and joint functioning in the individuals affected with stroke. FMA has excellent interrater and intrarater reliability and construct validity [12].

The Montreal Cognitive Assessment (MOCA) assesses the cognitive domains, namely attention, concentration, ex- ecutive functioning, orientation, language, visuospatial skills and memory through performance of various independent tasks. MOCA has acceptable responsiveness and criterion validity in stroke patients $[13,14]$.

The Wisconsin Gait Scale (WGS) constitutes a promising tool for a qualitative, observational analysis of gait in post-stroke individuals and allows for proper planning, monitoring and assessing rehabilitation results. WGS has a high internal consistency and test-retest reliability [15].

Stroke specific scale was used for objective measurement of gait and quality of life. Stroke Specific Quality of Life (SSQOL) measures the quality of life and is a standardized, reliable (reliability coefficient 0.92 ), validated scale that specifically measures the quality of life of individuals with stroke.

\section{Intervention}

The patient was intervened with M-tDCS over the left primary motor cortex PMC i.e. C3 point and left dorsolateral prefrontal cortex (L-DLPFC) i.e. F3 point with 0.5-2 mA intensity for a period of 20 minutes [12]. Points of stimulation were selected based on 10-20 electroencephalography international classification system. SaeboFlex assisted task oriented training $[16,17]$ FES over the LE to elicit dorsiflexion at the ankle and eversion of the foot and conventional physiotherapy rehabilitation including a tailored exercise program [18]. Whole intervention was given for five times per week for a total of 4 weeks.

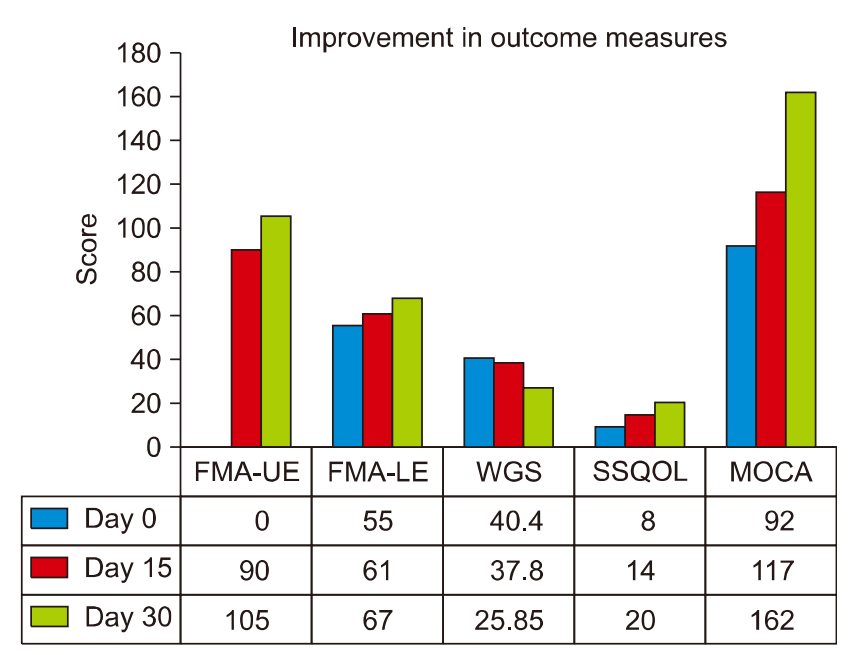

Figure 1. Improvement in outcome measures. FMA-UE: FuglMeyer assessment for upper extremity, FMA-LE: Fugl-Meyer assessment for lower extremity, WGS: Wisconsin gait scale, MOCA: Montreal cognitive assessment, SSQOL: stroke specific quality of life. 


\section{Results}

Mid and post-intervention assessment was done for all the outcome measures at day 15 and day 30 respectively in order to quantify the improvement of the patient. Patient improvement was observed in the motor and cognitive domains (Figure 1). Motor functions were found to be improved with the changes in the scores of FMA measured for the UE and LE. The FMA-UE score increased from 96 to 105 (Figure 2) and the FMA-LE scores increased from 55 to 67 (Figure 3). Similarly, improvement was also observed in the cognitive functions with a 10-point rise in MOCA scores from 8 to 18 (Figure 4). However, the patient still had difficulty in language comprehension and abstract thinking. The patient was ambulatory and was walking with the help of walker prior to the intervention. Improvement was also observed in his gait parameters with a reduction in WGS scores from 40.4 at day 0 to 25.85 at day 30 .

Improvement was also observed in the overall quality of life of the patient. His language, mobility, energy, social domains and UE functions were significantly better post intervention with overall changes in SSQOL scores from 92 to 162 over the period of 4 weeks (Figure 5). As reported by patient and his caregivers, they found decreased level of dependency for his activities of daily living and increased interest in participation in his community activities (Table 1).

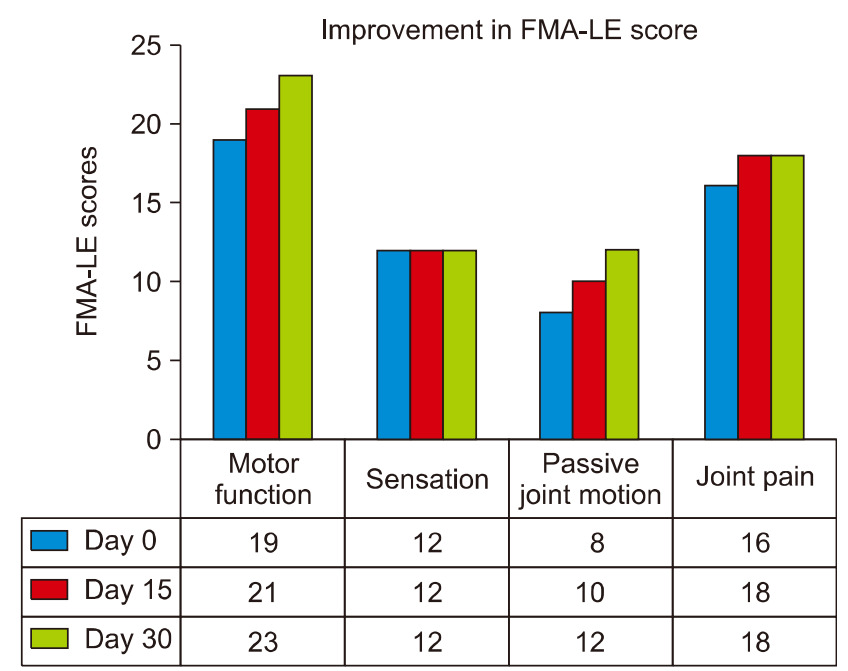

Figure 3. Improvement in Fugl-Meyer assessment for lower extremity (FMA-LE) score.
Figure 2. Improvement in Fugl-Meyer assessment for upper extremity (FMA-UE) score.
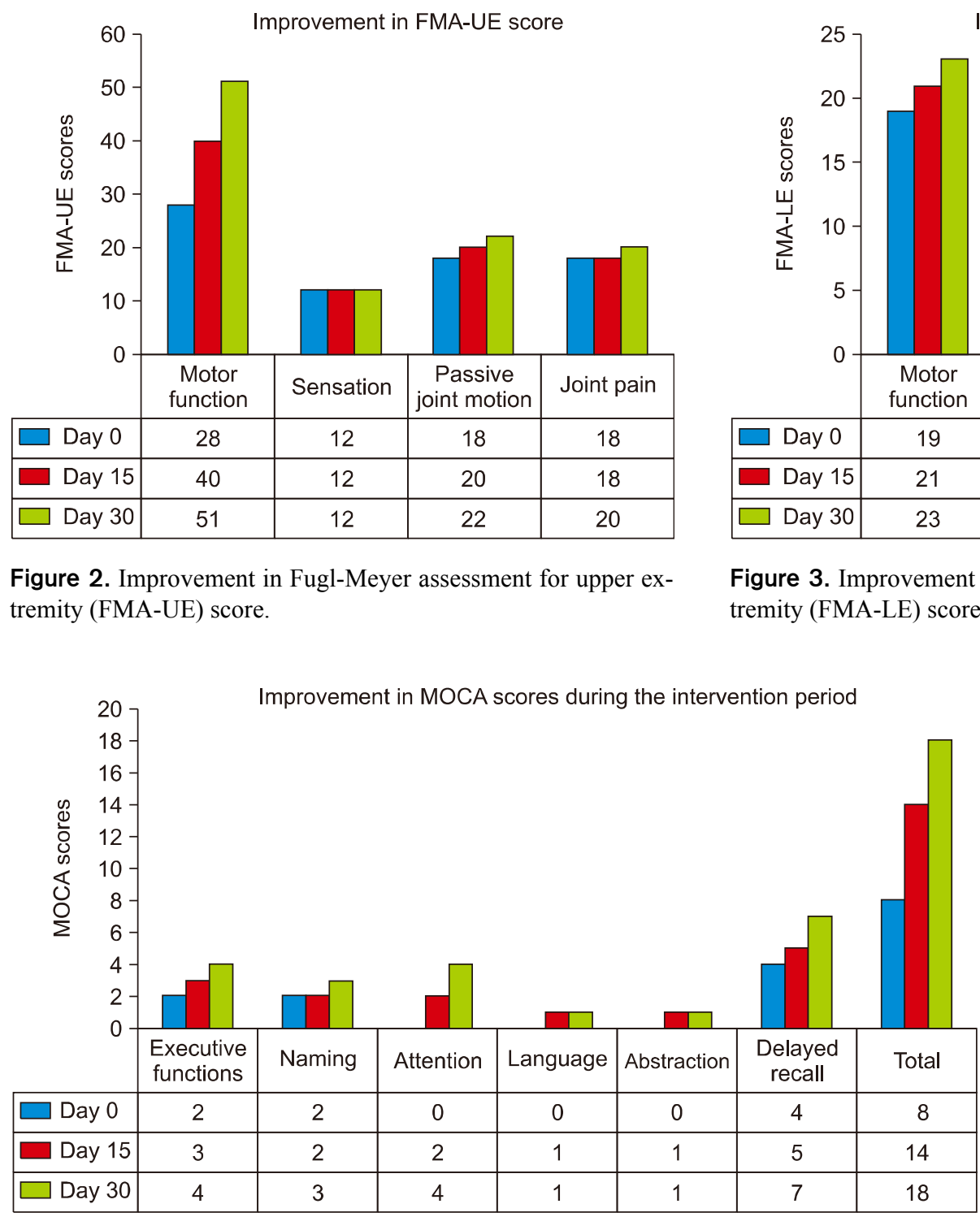

Figure 4. Improvement in Montreal cognitive assessment (MOCA) score. 


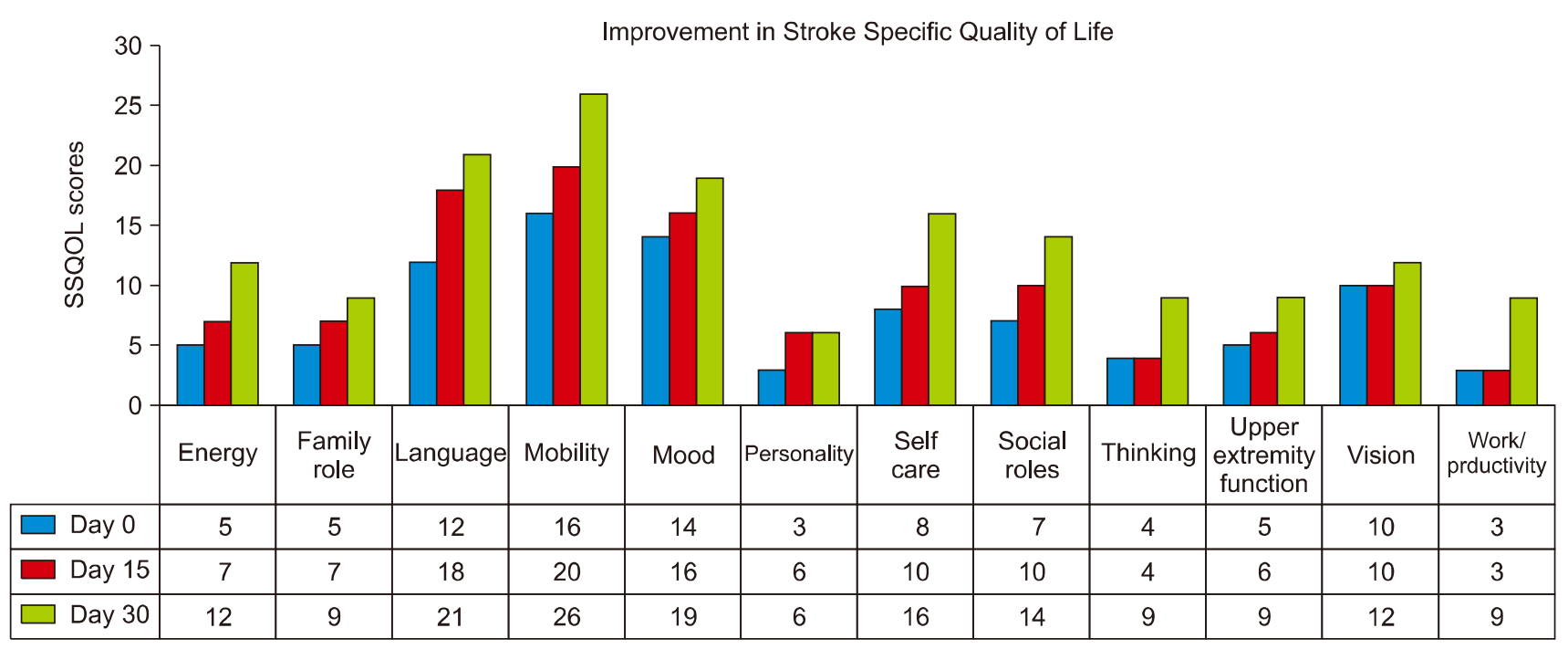

Figure 5. Improvement in Stroke Specific Quality of Life score.

Table 1. Improvement in FMA score during the intervention period

\begin{tabular}{lcccc}
\hline \multirow{2}{*}{ Outcome measure } & Maximum & \multicolumn{3}{c}{ Scores obtained } \\
\cline { 3 - 5 } & score & Day 0 & Day 15 & Day 30 \\
\hline FMA (UE) & 126 & 76 & 90 & 105 \\
FMA (LE) & 86 & 55 & 61 & 67 \\
WGS (score) & 42 & 40.4 & 37.8 & 25.85 \\
MOCA (score) & 30 & 8 & 14 & 20 \\
SSQOL (score) & 245 & 92 & 117 & 162 \\
\hline
\end{tabular}

FMA (UE): Fugl-Meyer assessment for upper extremity, FMA (LE): Fugl-Meyer assessment for lower extremity, WGS: Wisconsin gait scale, MOCA: Montreal cognitive assessment, SSQOL: stroke specific quality of life.

\section{Discussion}

Noninvasive brain stimulation is an emerging neuromodulation method to induce brain plasticity. Among such methods, transcranial magnetic stimulation and tDCS are most commonly used in the field of basic neuroscience and clinical application. But, the present work is discrete in its implementation of the M-tDCS along with SaeboFlex, FES and conventional rehabilitation for a period of 4 weeks determining their effect on overall recovery in a subacute stroke survivor.

The theoretical underpinning for clinical applicability of M-tDCS on individuals with stroke, targeting motor as well as DLPFC was developed from the fact that both motor as well as cognitive impairments coexist in stroke survivors causing devastating effects on their quality of life. The association between motor and cognitive impairment after stroke was explored in a review study. Authors of the study concluded that motor and cognitive impairments after stroke were correlated, despite the heterogeneity in populations, designs and tasks used in the studies included in this review. The most consistent finding was that the disturbances of gait, balance and limb function were often accompanied by deficits in attention and executive function [19].

The idea behind the use of peripheral stimulation in the form of SaeboFlex training and FES was to increase the magnitude of facilitatory effects induced by M-tDCS. Motor functions were significantly improved by application of tDCS with physical and occupational therapy in a study conducted by Hoyer et al. [9]. They reported the effect of the combination of peripheral nerve stimulation and tDCS on finger motor sequence task in persons with chronic stroke. Thus peripheral electrical stimulation might have an additional effect on the plastic change in the motor cortex induced by tDCS [20].

Results in the current patient have been found to be analogous to a study conducted on patients with Parkinson disease in which multitarget transcranial direct current stimulation of the primary motor cortex and left dorsolateral prefrontal cortex induced immediate after effects in the brain that translate into reduced freezing episodes of gait and improvements in executive function and mobility. Dagan et al. [16] facilitated motor cortex (M1) and the L-DLPFC by multichannel transcranial stimulation in comparison with stimulation of M1 only and a sham condition. Multitarget stimulation of both areas provided a significant improvement over the other conditions. To the best of our knowledge, 
there is huge dearth of evidence regarding clinical utility of M-tDCS in stroke participants. The present case report can be an important support as a window of opportunity for conducting large sample trials in order to establish the quality of evidence. This single participant case study provided us with vital evidence for M-tDCS in subacute stroke survivors though a larger trial shall suffice the quality of evidence.

\section{Limitations}

The findings of this study cannot be generalized since this is a single participant study and no comparison has been done in order to establish the efficacy of M-tDCS. However, an integrated approach of the M-tDCS has provided the add-on effect with respect to the improvement in motor as well as cognitive domains of the patient. Large sample trials are required to establish the effectiveness of M-tDCS in stroke survivors.

To conclude, the application of M-tDCS induced improvements in motor functions of the UE and LE, gait parameters and cognitive functions of the patient. The participant became an independent community ambulatory at a speed at least $50 \%$ of normal. This evidence will be crucial in designing better regimen, both in terms of content and context, of the rehabilitation process after stroke.

\section{Acknowledgements}

Department of Physiotherapy, Punjabi University for resource allocation and participant recruitment. Equipment support by Medicaid India.

\section{Conflict of Interest}

The authors declared no potential conflicts of interest with respect to the authorship and/or publication of this article.

\section{References}

1. Kamalakannan S, Gudlavalleti ASV, Gudlavalleti VSM, Goenka $\mathrm{S}$, Kuper H. Incidence \& prevalence of stroke in India: a systematic review. Indian J Med Res 2017;146:175-85.

2. Langhorne P, Bernhardt J, Kwakkel G. Stroke rehabilitation. Lancet 2011;377:1693-702.

3. Feigin VL, Norrving B, Mensah GA. Global burden of stroke. Circ Res 2017;120:439-48.

4. Gund BM, Jagtap PN, Ingale VB, Patil RY. Stroke: a brain attack. IOSR J Pharm 2013;3(8 Pt 2):1-23.
5. Aqueveque P, Ortega P, Pino E, Saavedra F, Germany E, Gómez B. After stroke movement impairments: a review of current technologies for rehabilitation. In: Tan U, editor. Physical disabilities therapeutic implications. Rijeka: IntechOpen; 2017.

6. Alon G, Levitt AF, McCarthy PA. Functional electrical stimulation enhancement of upper extremity functional recovery during stroke rehabilitation: a pilot study. Neurorehabil Neural Repair 2007;21:207-15.

7. Leon D, Cortes M, Elder J, Kumru H, Laxe S, Edwards DJ, et al. tDCS does not enhance the effects of robot-assisted gait training in patients with subacute stroke. Restor Neurol Neurosci 2017; 35:377-84.

8. Goodwill AM, Teo WP, Morgan P, Daly RM, Kidgell DJ. Bihemispheric-tDCS and upper limb rehabilitation improves retention of motor function in chronic stroke: a pilot study. Front Hum Neurosci 2016;10:258.

9. Hoyer EH, Celnik PA. Understanding and enhancing motor recovery after stroke using transcranial magnetic stimulation. Restor Neurol Neurosci 2011;29:395-409.

10. Guan YZ, Li J, Zhang XW, Wu S, Du H, Cui LY, et al. Effectiveness of repetitive transcranial magnetic stimulation (rTMS) after acute stroke: a one-year longitudinal randomized trial. CNS Neurosci Ther 2017;23:940-6.

11. Bao SC, Khan A, Song R, Kai-Yu Tong R. Rewiring the lesioned brain: electrical stimulation for post-stroke motor restoration. J Stroke 2020;22:47-63.

12. Gladstone DJ, Danells CJ, Black SE. The fugl-meyer assessment of motor recovery after stroke: a critical review of its measurement properties. Neurorehabil Neural Repair 2002;16:232-40.

13. Wu CY, Hung SJ, Lin KC, Chen KH, Chen P, Tsay PK. Responsiveness, minimal clinically important difference, and validity of the MoCA in stroke rehabilitation. Occup Ther Int 2019;2019:2517658.

14. Chiti G, Pantoni L. Use of Montreal Cognitive Assessment in patients with stroke. Stroke 2014;45:3135-40.

15. Guzik A, Drużbicki M, Przysada G, Kwolek A, BrzozowskaMagoń A, Wyszyńska J, et al. Assessment of test-retest reliability and internal consistency of the Wisconsin Gait Scale in hemiparetic post-stroke patients. Adv Rehabil 2016;30:41-53.

16. Dagan M, Herman T, Harrison R, Zhou J, Giladi N, Ruffini G, et al. Multitarget transcranial direct current stimulation for freezing of gait in Parkinson's disease. Mov Disord 2018;33:642-6.

17. Franck JA, Timmermans AAA, Seelen HAM. Effects of a dynamic hand orthosis for functional use of the impaired upper limb in sub-acute stroke patients: a multiple single case experimental design study. Technol Disabil 2013;25:177-87.

18. Woo Y, Jeon H, Hwang S, Choi B, Lee J. Kinematics variations after spring-assisted orthosis training in persons with stroke. Prosthet Orthot Int 2013;37:311-6.

19. Sabut SK, Sikdar C, Mondal R, Kumar R, Mahadevappa M. Restoration of gait and motor recovery by functional electrical stimulation therapy in persons with stroke. Disabil Rehabil 2010;32:1594-603.

20. Verstraeten SMM, Mark RE, Sitskoorn MM. Motor and cognitive impairment after stroke: a common bond or a simultaneous deficit? Stroke Res Ther 2016;1:1. 